\title{
From fusion to total disassembly: global stopping in heavy-ion collisions
}

\author{
Jatinder K. Dhawan, Narinder Dhiman, Aman D. Sood, and Rajeev K. Puri \\ Department of Physics, Panjab University, Chandigarh-160 014, India.
}

December 8, 2018

\begin{abstract}
Using the quantum molecular dynamics model, we aim to investigate the emission of light complex particles, and degree of stopping reached in heavy-ion collisions. We took incident energies between 50 and $1000 \mathrm{MeV} /$ nucleon. In addition, central and peripheral collisions and different masses are also considered. We observe that the light complex particles act in almost similar manner as anisotropic ratio. In other words, multiplicity of light complex particles is an indicator of global stopping in heavy-ion collisions. We see that maximum light complex particles and stopping is obtained for heavier masses in central collisions.
\end{abstract}

Electronic address: rkpuri@pu.ac.in 
The study of nuclear reactions from low to relativistic energies provides variety of phenomena. At low energies, Pauli principle blocks any significant scattering of the nucleons. Therefore, attractive mean field dominates the physics in this energy regime [1]. At intermediate energies, however, a mixture of attractive mean field and repulsive nucleonnucleon scattering exists $[2,3]$. Both these regimes together, lead the matter from a fused state to total disassembly. One is also interested to understand the mechanism behind this. Further, the origin of small pieces (fragments) is also of great interest. One is trying to correlate this origin with global stopping [4]. The degree of stopping however, may vary drastically with incident energies, mass of colliding nuclei and colliding geometry. The degree of global stopping has also been linked with the thermalization (equilibrium) in heavy-ion collisions.

Theoretically, these happenings are followed by a variety of models. Some models assume a priori equilibrium (at least at local level) whereas others hunt for the degree of thermalization in a reaction. Several models which depend on the assumption of equilibrium, have been applied successfully to study the physics at low and intermediate energies $[5,6]$. At the same time, the light and medium mass fragments (produced and emitted in reactions), have also been used to get information about the thermalization and stopping in heavy-ion collisions $[4,7]$. The origin of light and medium mass fragments is still under debate $[3,4]$.

We here plan to investigate the degree of stopping reached in these reactions from fusion to total disassembly. We shall also attempt to correlate the emission of light mass fragments and degree of stopping reached in a reaction. A complete knowledge about the degree of stopping is very important since it can be connected to the properties of the system equation of states and in medium properties of the nucleon-nucleon cross section [8]. Our study is based on the analysis of reactions between 50 and $1000 \mathrm{MeV} /$ nucleon. Our aim is to look for the randomization of one-body momentum space or memory loss of the incoming momentum. This is also termed as global stopping in the literature. Sometimes, this randomization is also related to the dynamical thermalization of the nuclear matter in heavy-ion collisions. For this analysis, we employ the quantum molecular dynamics (QMD) model. The reader is referred to Ref.(3) for details.

For the present analysis, thousands of events were simulated for the reactions of ${ }^{20} \mathrm{Ne}+{ }^{20} \mathrm{Ne},{ }^{40} \mathrm{Ca}+{ }^{40} \mathrm{Ca},{ }^{58} \mathrm{Ni}+{ }^{58} \mathrm{Ni},{ }^{93} \mathrm{Nb}+{ }^{93} \mathrm{Nb},{ }^{139} \mathrm{La}+{ }^{139} \mathrm{La}$ and ${ }^{197} \mathrm{Au}+{ }^{197} \mathrm{Au}$ between 50 and $1000 \mathrm{MeV} /$ nucleon using hard equation of state along with free energy dependent nucleon-nucleon cross-section. The geometrical choice is varied between the most central to peripheral ones. This large choice will give us possibility to look for the matter from the fusion to complete disassembly. The choice of symmetric reactions is to avoid any influence of the asymmetry of colliding nuclei [9]. As reported in Ref. [9], the asymmetric nuclei have quite different picture compared to symmetric nuclei. One should keep in the mind that at low energies, one has pre-equilibrium emission of particles from excited compound nucleus.

The colliding nuclei not only compress each other, they also heat the matter $[4,10]$. In addition, the destruction of initial correlations, makes the matter homogenous and one can have global stopping. More the initial memory of nucleons is erased, better it is stopped and better one has average mixing of projectile and target momentum. We shall here consider few different quantities capable of estimating the degree of global stopping. 
The first quantity is the anisotropy ratio $<R_{a}>$ which is defined as $[10,11]$ :

$$
\left\langle R_{a}\right\rangle=\frac{\sqrt{\left\langle p_{x}^{2}\right\rangle}+\sqrt{\left\langle p_{y}^{2}\right\rangle}}{2 \sqrt{\left\langle p_{z}^{2}\right\rangle}} .
$$

This anisotropy ratio $\left\langle R_{a}\right\rangle$ is an indicator of the global stopping and randomization of momentum of target and projectile of the system. The global word is due to the fact that it does not depend on the local position. Naturally for a complete mixing, $\left\langle R_{a}\right\rangle$ ratio should be close to unity. Alternatively, in the literature, $\left\langle E_{r a t}\right\rangle\left(=\frac{\sum p_{\perp}^{2} / 2 m n}{\sum p_{z}^{2} / 2 m n}\right)$ has also been proposed for the mixing. There maximum value of $\left\langle E_{r a t}\right\rangle$ was supposed to be equal to $2[4]$.

The second possibility for the degree of stopping of nuclear matter is to look for the rapidity distribution which is defined as

$$
Y(i)=\frac{1}{2} \ln \frac{\mathbf{E}(i)+\mathbf{p}_{z}(i)}{\mathbf{E}(i)-\mathbf{p}_{z}(i)},
$$

where $\mathbf{E}(i)$ and $\mathbf{p}_{z}(i)$, are, respectively, the total energy and longitudinal momentum of ith particle. Naturally, for a complete stopping, one should expect a single Guassian shape of the rapidity. Very often, the nature of emitting source is defined by analyzing the rapidity distribution.

Apart from the global stopping and randomization of phase-space, one may also define the local average mixing of target and projectile. Here, one studies, the relative momentum of two colliding Fermi spheres which indicates the deviation from a single Fermi sphere and by that from mixing. The concept of local stopping is used by the hydrodynamical models to simulate the heavy-ion reactions. All these definitions will give us a glimpse of the degree of stopping or mixing (and sometimes of equilibrium) reached in a reaction. We shall also work out its relation with the production of light complex particles (LCP's) defined as fragments with $(2 \leq A \leq 4)$. Here the light complex particles are detected with two different methods: In the first approach, one binds nucleons if they are within a distance of $4 \mathrm{fm}$. This method is labelled as minimum spanning tree (MST) method. This method, by virtue, of it's definition may miss the light complex particles emitted at a later stage of the reaction either from quasi target/projectile or fusion residue. To cope with this, we also employ the sophisticated simulated annealing clusterization algorithm (SACA) as a second approach, which is based on the idea of obtaining that fragment's structure which maximizes the binding energy of the system [12]. This algorithm is based on the Metropolis algorithm and has been reported to explain the data throughout the energy range [12].

In Fig. 1, we display the final phase-space of a single event of ${ }^{197} \mathrm{Au}+{ }^{197} \mathrm{Au}$ at 400 $\mathrm{MeV} /$ nucleon. The different panel are at $\mathrm{b}=0,3$ and $6 \mathrm{fm}$, respectively. Here only LCP's and IMF's $(5 \leq A \leq 65)$ are displayed. We note that the central collisions lead to a complete spherical distribution. This degree of stopping decreases with the increase in impact parameter. Further the light complex particles mostly originate from the mid-rapidity region. In other words, LCP's can also act as a barometer for studying the stopping in heavy-ion collisions. The IMF's, however, are pointing towards either 

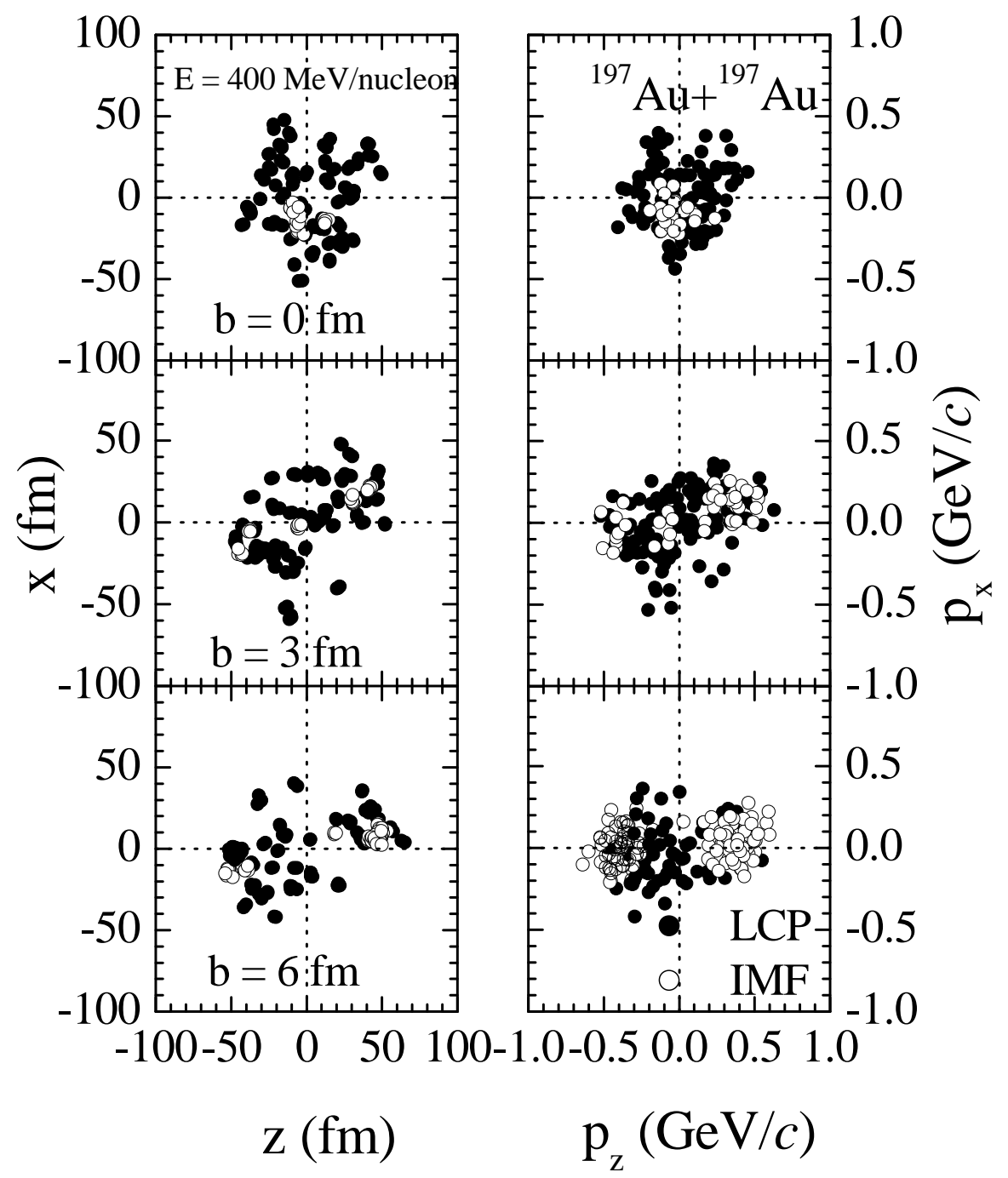

Figure 1: The snap-shots of a single event in the phase-space. The top, middle, and bottom panels are, respectively, for impact parameters $b=0,3$, and $6 \mathrm{fm}$. The filled circles represent the LCP's and the open circles represent IMF's. 


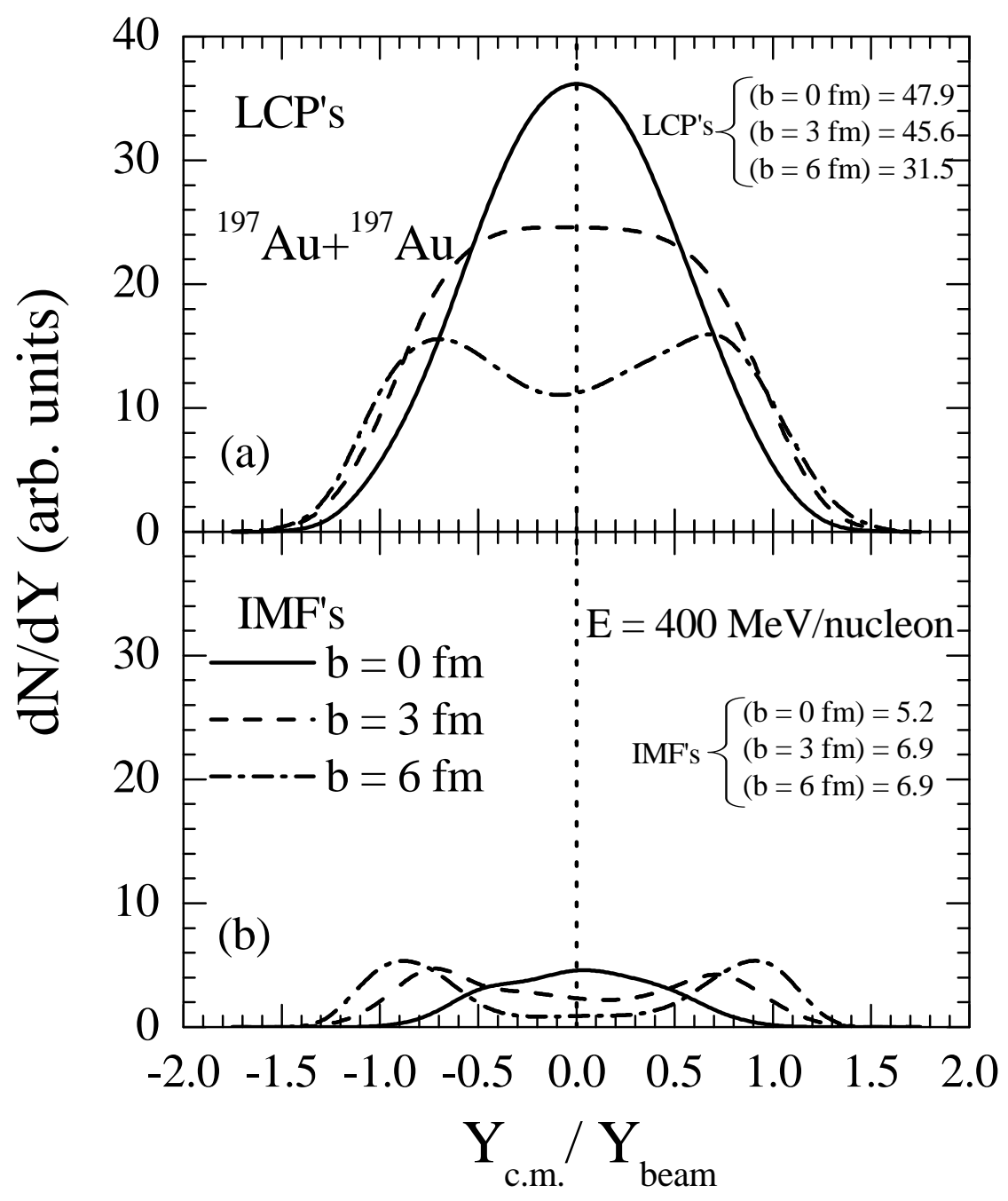

Figure 2: The rapidity distribution (of LCP's and IMF's) $\frac{d N}{d Y}$ as a function of reduced rapidity. The reaction is of ${ }^{197} \mathrm{Au}+{ }^{197} \mathrm{Au}$ at an incident energy of $400 \mathrm{MeV} /$ nucleon. 


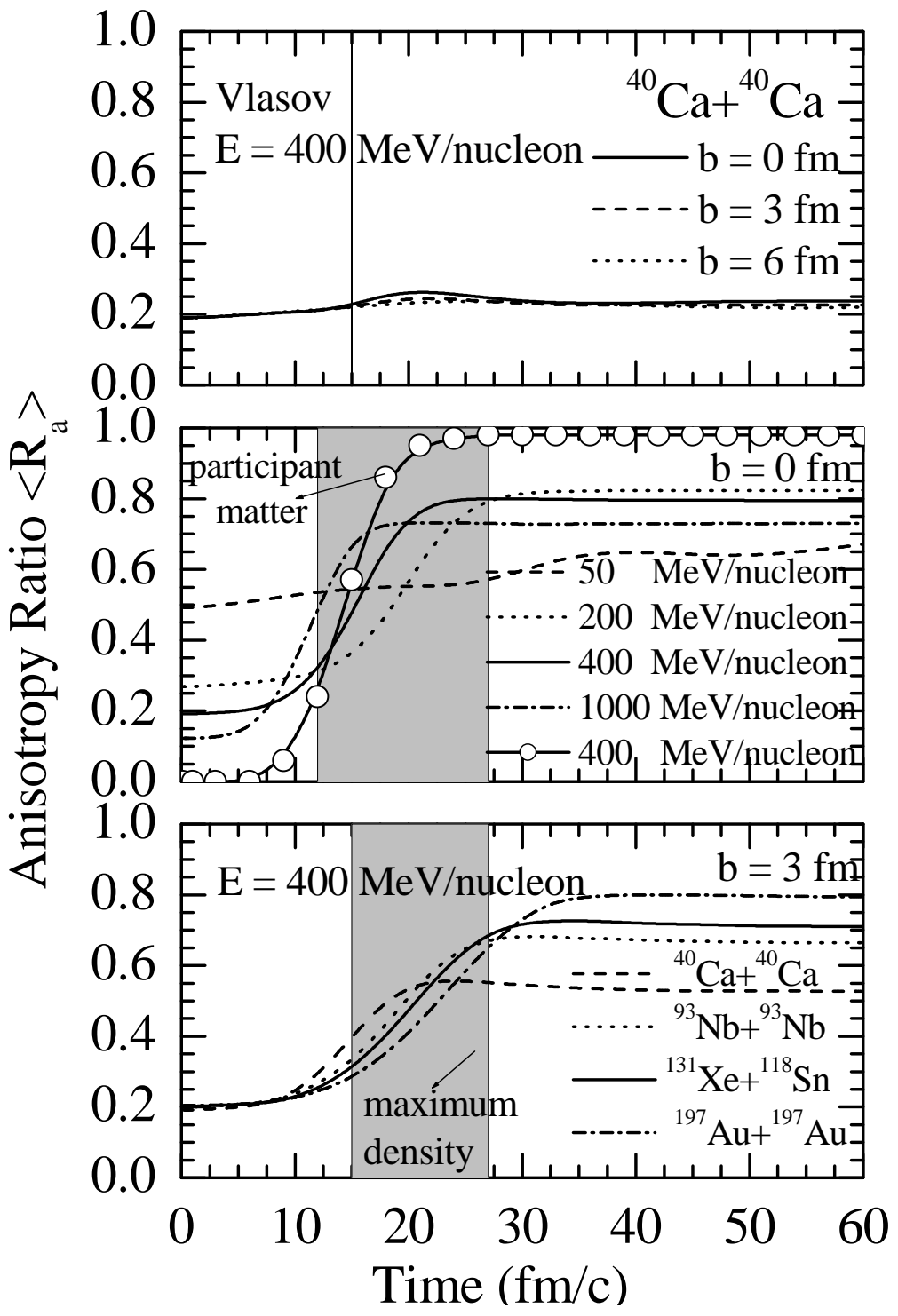

Figure 3: The time evolution of anisotropy ratio $\left\langle R_{a}\right\rangle$ is displayed. The upper panel is for ${ }^{40} \mathrm{Ca}+{ }^{40} \mathrm{Ca}$ at an incident energy of $400 \mathrm{MeV} /$ nucleon in the Vlasov mode. The middle panel is for ${ }^{40} \mathrm{Ca}+{ }^{40} \mathrm{Ca}$ at different incident energies. The lower panel is for ${ }^{40} \mathrm{Ca}+{ }^{40} \mathrm{Ca}$, ${ }^{93} \mathrm{Nb}+{ }^{93} \mathrm{Nb},{ }^{131} \mathrm{Xe}+{ }^{118} \mathrm{Sn}$ and ${ }^{197} \mathrm{Au}+{ }^{197} \mathrm{Au}$, respectively. 


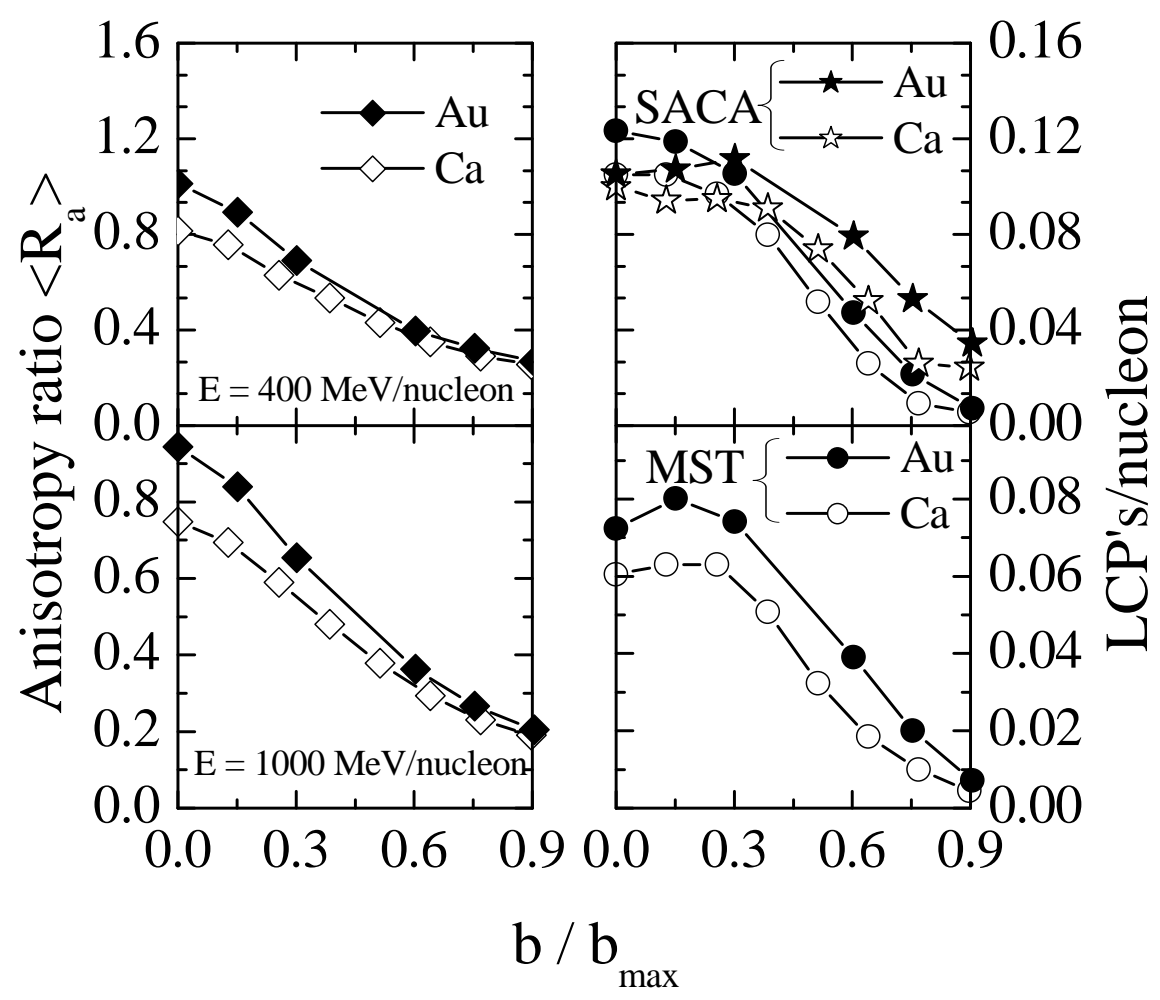

Figure 4: The anisotropic ratio $\left\langle R_{a}\right\rangle$ and LCP's/nucleon as a function of normalized impact parameter. The reactions considered are of ${ }^{40} \mathrm{Ca}+{ }^{40} \mathrm{Ca}$ and ${ }^{197} \mathrm{Au}+{ }^{197} \mathrm{Au}$. The upper panel is at $\mathrm{E}=400 \mathrm{MeV} /$ nucleon whereas lower panel is at $\mathrm{E}=1000 \mathrm{MeV} /$ nucleon. Here LCP's are obtained within MST and SACA approaches.

target or projectile regions. Therefore, these are originating from the surface of colliding nuclei. In other words, these can be viewed as remnant of the spectator matter. It has been discussed by many authors that the intermediate mass fragments carry the initial memory of nuclei and correlations. Naturally, one can not expect them to be emitted from mid-rapidity region. Since degree of spectator matter increase with impact parameter, so is the formation of either IMF's or heavier fragments. We have also studied a large number of different individual events. The above analysis is quite similar in all these events.

To further quantify this observation, we display in Fig. 2, the rapidity distribution $\mathrm{dN} / \mathrm{dY}$ for LCP's and IMF's. We see that the LCP's emitted in central collisions are originating from a complete stopped source. The central collisions are better randomized compared to peripheral ones. The conclusions here match with the Fig.1 and also in agreement with earlier reports $[4,6,7]$. It is worth mentioning that the Gaussian shape depends on the size of colliding nuclei. For lighter colliding nuclei, it is more of a flat distribution whereas for heavier nuclei, it is more of a Gaussian type [9].

In Fig. 3 we display the global stopping and randomization in terms of anisotropic ratio $\left\langle R_{a}\right\rangle$ in the mass-energy-impact parameter plane. If we don't allow the nucleon-nucleon collisions to happen (i.e. in a Vlasov mode), matter does not randomize globally at all. 
It is argued by many authors that the role of mean field is independent of mass of the colliding system. Here we show that it is also independent of the impact parameter. One notices that even at small incident energies (e.g. $50 \mathrm{MeV} /$ nucleon), $60 \%$ randomization can be achieved. However, at very high incident energy (e.g. $1 \mathrm{GeV} /$ nucleon), system does not advance towards global randomization. Instead, a decrease in the degree of stopping points toward transparency in nuclear matter with increase in incident energy. This is also in agreement with Ref. [4].

Looking at the bottom panel, one also notices a linear trend for the mass dependence of anisotropic ratio $\left\langle R_{a}\right\rangle$. At a fixed impact parameter, heavier colliding nuclei are better randomized compared to lighter colliding nuclei. We will come back to this point later on. This conclusion is independent of the mass of colliding nuclei, impact parameter as well as incident energy. From the Figure, one also notices that anisotropic ratio $\left\langle R_{a}\right\rangle$ changes drastically during high density phase $\left(\rho>\rho_{0}\right)$. Once high density phase is over, no more changes occur in the thermalization. In other words, the nucleon-nucleon collisions happening after the high density phase do not produce substantial changes. It is worth mentioning that collective transverse flow also saturates once high density phase is over. Further, as shown in the Figure, participant matter (nucleons suffering at least one collision) also follows the same trend. It means the nucleons going under nucleon-nucleon collisions after high density phase do not play a role. We shall now attempt to correlate the emission of LCP's and anisotropic ratio $\left\langle R_{a}\right\rangle$. As noted, both these are the indicator of global stopping and randomization of momentum-space in heavy-ion collisions.

In Fig. 4, we display $\left\langle R_{a}\right\rangle$ ratio and LCP's/nucleon (obtained in MST and SACA approaches) as a function of the colliding geometry. Both $\left\langle R_{a}\right\rangle$ ratio and LCP's/nucleon decrease (almost in same fashion) with increase in impact parameter. Further the mass dependence has a little role to play. A change of mass from 40 units to 400 units does not yield significant changes in both the case. The same trend at both incident energies points toward linear relation between both these quantities. As stated earlier, the emission of LCP's originate from a complete randomized source. It is also interesting to note that the trend of LCP's/nucleon is similar in both MST and SACA methods pointing towards complete emission of LCP's in these reactions. The decrease in LCP's complements corresponding increase in IMF's and heavy fragments. These fragments are the remanent of spectator matter which is non-stopped. Therefore, a decrease in LCP's multiplicity directly measures a decrease in the degree of randomization and hence global stopping. Therefore, there is one to one correspondence between both these quantities.

In Fig. 5 we display the mass dependence of $\left\langle R_{a}\right\rangle$ ratio and LCP's*/nucleon (obtained in both MST and SACA approaches) at $400 \mathrm{MeV} /$ nucleon. Here, LCP's*/nucleon are scaled values of LCP's/nucleon by a factor of 6 (i.e. LCP's/nucleon*6). We see that $\left\langle R_{a}\right\rangle$ ratio has a little mass dependence. Also, LCP's*/nucleon in both approaches has similar mass dependence. The mass dependence in both the cases can be parameterized by a power law $\propto A^{\tau}$ with $\tau$ close to $0.1,0.07$ and 0.09 respectively. A very similar impact parameter and mass dependence of anisotropy ratio and LCP's*/nucleon clearly demonstrate that the LCP's production is closely related to the global randomization in heavy-ion collisions.

As seen in Fig. 1, the LCP's are emitted from mid-rapidity region where initial correlation and memory of nucleons is completely destroyed. The $\left\langle R_{a}\right\rangle$ ratio is also a 


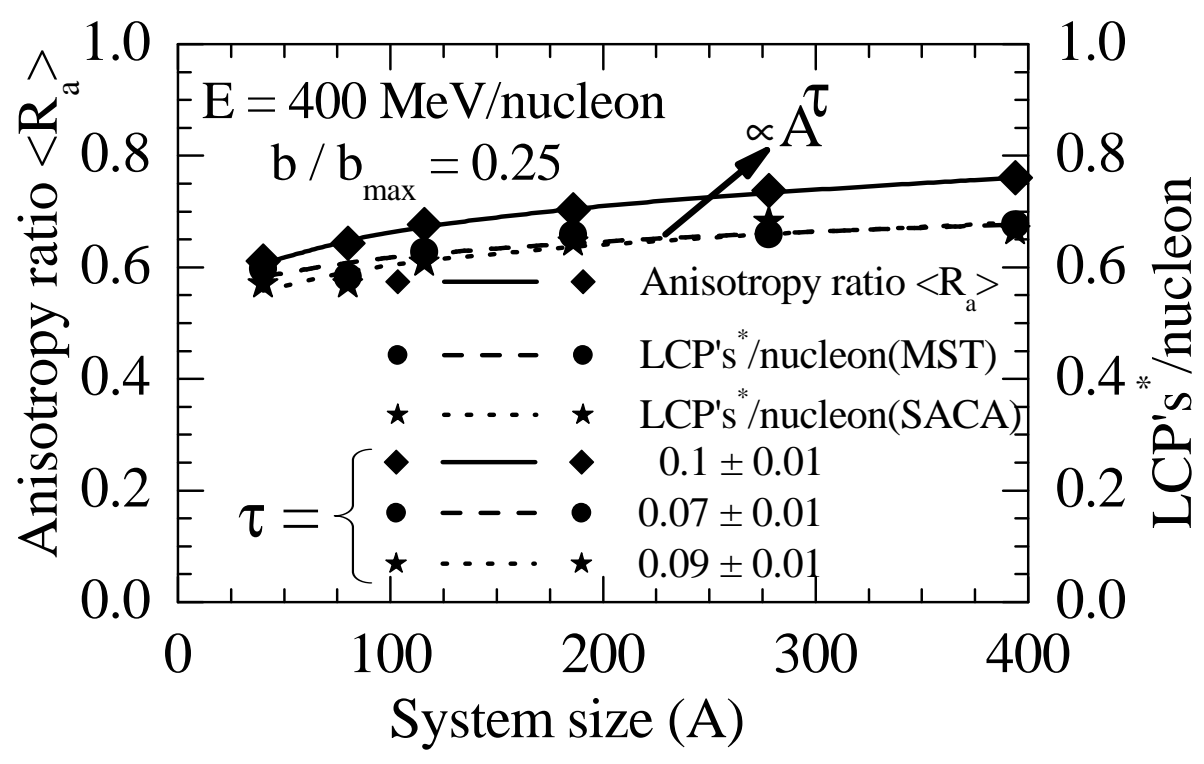

Figure 5: The anisotropy ratio $\left\langle R_{a}\right\rangle$ and LCP's* $/$ nucleon as a function of composite mass of colliding nuclei. Here we consider ${ }^{20} \mathrm{Ne}+{ }^{20} \mathrm{Ne},{ }^{40} \mathrm{Ca}+{ }^{40} \mathrm{Ca},{ }^{58} \mathrm{Ni}+{ }^{58} \mathrm{Ni},{ }^{93} \mathrm{Nb}+{ }^{93} \mathrm{Nb}$, ${ }^{139} \mathrm{La}+{ }^{139} \mathrm{La}$ and ${ }^{197} \mathrm{Au}+{ }^{197} \mathrm{Au}$ reactions.

direct indicator of breaking the initial correlations and erasing the memory of nucleons. We see that the geometrical and mass behavior is quite similar in both cases. Therefore, both, in principle are, general quantities to study the global stopping. In addition, one also notices that best randomization and stopping is obtained in central collisions for heavier masses. If one takes impact parameter averaged randomization, on notices that the this is close to 0.6. One can say that no global stopping is reached in inclusive heavy-ion collisions.

In summary, using quantum molecular dynamics model, we investigate the emission of light complex particles, and the degree of stopping reached in heavy-ion collisions. We observed that the light complex particles act in almost similar manner as anisotropic ratio. In other words, multiplicity of light complex particles production is an indicator of randomization in heavy-ion collisions. We see that maximum light complex particles and randomization is obtained for heavier mass at central collisions. The smaller mass and larger impact parameters lead to less equilibrated matter.

The work is supported by Department of Atomic Energy, Government of India, India.

\section{References}

[1] L. C. Vaz et al., Phys. Rep. 69, 373 (1981); R. K. Puri, Ph. D. Thesis, Panjab University, Chandigarh (1990).

[2] H. Stöcker, and W. Greiner, Phys. Rep. 137, 277 (1986). 
[3] J. Aichelin, Phys. Rep. 202, 233 (1991); C. Hartnack et al., Eur. Phys. J A 1, 151 (1998).

[4] P. B. Gossiaux and J. Aichelin, Phys. Rev. C 56, 2109 (1997).

[5] J. P. Bondorf et al., Nucl. Phys. A443, 321 (1985); D. H. E. Gross, Rep. Prog. Phys. 53, 605 (1990).

[6] L. G. Moretto and G. J. Wozniak, Annu. Rev. Nucl. Part. Sci. 43, 379 (1993).

[7] J. Lukasik et al., Phys. Rev. C 55, 1906 (1997); ibid, Phys. Rev. C 61, 014606 (1997); ibid, Phys. Rev. C 66, 064606 (2002); T. Lefort et al., Nucl. Phys. A602, 397 (2000); F. Rami et al., Phys. Rev. Lett. 84, 1120 (2000); W. Reisdorf et al., Phys. Rev. Lett. 92, 232301 (2004).

[8] B. Hong et al., Phys. Rev. C 71, 034902 (2005); T. Gaitanos, C. Fuchs and H. H. Wolter, Phys. Lett. B 609, 241 (2005); P. Danielewicz, Acta Phys. Polon. B 33, 45 (2002).

[9] J. Singh, S. Kumar and R. K. Puri, Phys. Rev. C 62, 044617 (2000); ibid, Phys. Rev. C 65, 024602 (2002).

[10] D. T. Khoa et al., Nucl. Phys. A542, 671 (1992); R. K. Puri et al., Nucl. Phys. A575, 733 (1994); D. Hahn and H. Stöcker, Nucl. Phys. A476, 718 (1988).

[11] R. K. Puri et al., J. Phys. G: Nucl. Part. Phys. 20, 1817 (1994).

[12] R. K. Puri et al., Phys. Rev. C 54, R28 (1996). 